\title{
The CME dynamics associated to the prominence eruption of December 2, 2003
}

\author{
Vladimir A. Slemzin ${ }^{1}$, S. Koutchmy ${ }^{2}$, J.-C. Noens ${ }^{3}$ and D. Romeuf ${ }^{4}$ \\ ${ }^{1}$ P.N. Lebedev Physical Institute, Lenisky pr., 53, Moscow, 119991, Russia \\ email: slem@lebedev.ru \\ ${ }^{2}$ Institut d'Astrophysique de Paris, CNRS and UPMC, France \\ email: koutchmy@iap.fr \\ ${ }^{3} \mathrm{OMP}$ and Pic du Midi observatory, France \\ email: noens@ast.obs-mip.fr \\ ${ }^{4}$ C.R.I. Claude Bernard Lyon I University, O.A.-Fiducial, France \\ email: David.Romeuf@recherche.univ-lyon1.fr
}

\begin{abstract}
We report on a complex study of a typical large W-limb CME event occurring on December 2, 2003 in the vicinity of AR 0508. It is associated with a prominence eruption which has been observed with the Pic du Midi Ha flux coronagraph as well as in EUV by both the SOHO/EIT and the Coronas/SPIRIT space telescopes. The eruption started with the emergence of a fast expanding loop between 9 and 10 UT, followed by a heating and an acceleration of the erupted material and resulted in three-part CME observed by LASCO after 10:50 UT. A temporal analysis of the prominence motion and the EUV dimming light curve have shown that both the frontal structure and the core of a CME were initiated simultaneously with the peak of the X-ray C7.2 flare. The total mass of the prominence including the $\mathrm{H} \alpha$ filament and EUV filament channel is close to the mass of the core but significantly less than the total CME mass.
\end{abstract}

Keywords. Sun: corona, Sun: coronal mass ejections (CMEs)

We report on a large W-limb event, which occurred on December 2, 2003, 9-14h (hereafter UT), in the vicinity of an old multi-polar region 10508 . The prominence eruption was observed in $\mathrm{H} \alpha$ with the HACO coronagraph, in EUV with SOHO/EIT and CORONASF/SPIRIT telescopes. The associated CME was observed later with the LASCO C2 and C3 white-light coronagraphs. Two weeks before a large two-part filament was seen to the North of NOAA AR 10508 in $\mathrm{H} \alpha$ as well as in EUV (EUV filament channel - EFC). In $\mathrm{H} \alpha$ the filament has a full length of $250 \mathrm{Mm}$, a width - $10 \mathrm{Mm}$ and a volume $2 \cdot 10^{28} \mathrm{~cm}^{3}$. Following Heinzel et al. (2003), for measured $\tau \sim 1$ we estimated the neutral hydrogen density $\mathrm{n}_{1}=1.6 \cdot 10^{10} \mathrm{~cm}^{-} 3$, electron $\left(\mathrm{H}\right.$-ion) density $\mathrm{n}_{e}=1.7 \cdot 10^{10} \mathrm{~cm}^{-3}$, and the mass of the $\mathrm{H} \alpha$ filament $\mathrm{M}_{H}=2 \cdot 10^{15} \mathrm{~g}$. The total mass including the EFC (Aulanier\&Schmieder 2002, Heinzel et al. 2003) was then $\mathrm{M}_{F}=3-4 \cdot 10^{15} \mathrm{~g}$.

On December 2 after 09:10 the prominence was seen in $\mathrm{H} \alpha$ with the HACO coronagraph at Pic du Midi observatory (Romeuf et al. 2006) as a luminous object due to UV and EUV excitation of hydrogen by radiation of corona and chromosphere (Henzel\&Rompolt, 1987). At 09:13 a new fast expanding loop emerged in the center of AR 508 and destabilized the prominence. Fig. 1 shows the most important stages of this process seen in HACO (a-c) and EIT $195 \AA$ (d-f) as well as integrated light curves in $\mathrm{H} \alpha$ (in the $250-270^{\circ}$ sector), $195 \AA$ (the same sector) and in X-rays (GOES). The brightness of the prominence first increased in $\mathrm{H} \alpha$ (maximum at 09:35) due to growth of turbulence (see Gontikakis et al. 1997), then decreased in $\mathrm{H} \alpha$ and increased locally in EUV due to heating process. At 09:48 (which coincides with the maximum of the C7.2 flare) the 

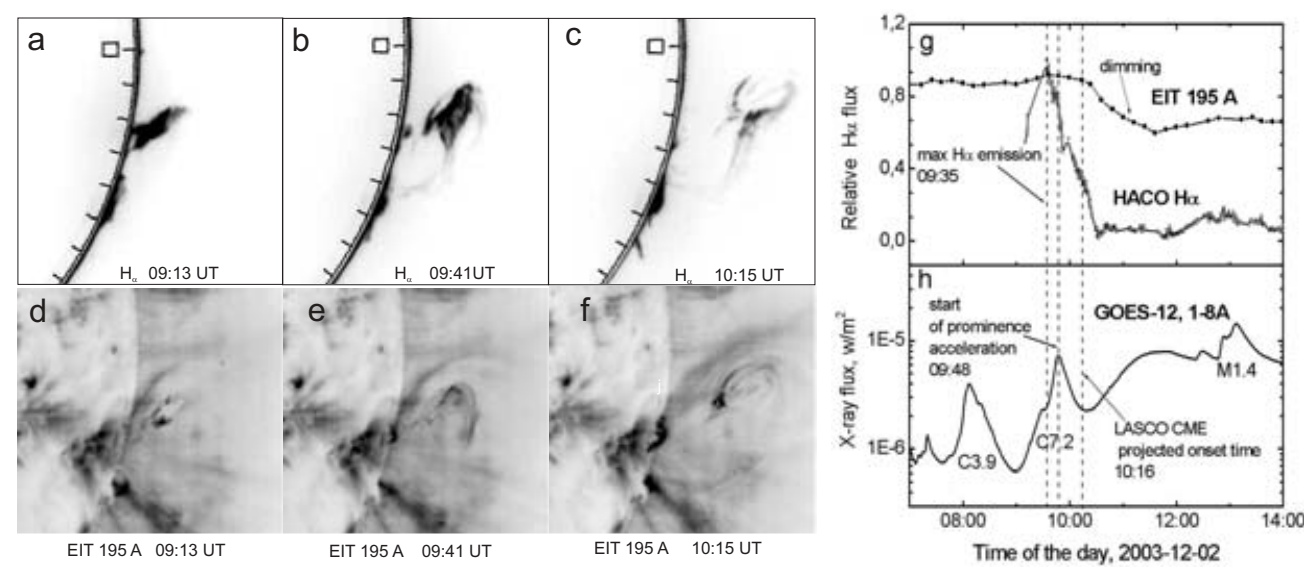

Figure 1. Dynamics of eruptive prominence of December 2, 2003. (a-c) H $\alpha$ HACO images. (d-f) EIT $195 \AA$ images at the same times. (g) Total brightness light curves in $\mathrm{H} \alpha$ and EUV $195 \AA$ integrated over the sector $250-270^{\circ}$. (h) X-ray GOES flux

prominence began to accelerate and move outward with the hottest part directed to the Sun.

The three-part CME appeared in the LASCO C2 after 10:50. As a result of the CME development, the streamer seen before at the periphery of the region, deflected to the South, and its brightness increased. At the distances from 4 to $25 \mathrm{R}_{\odot}$ the frontal structure moved with a near constant velocity of $1393 \mathrm{~km} / \mathrm{c}$, the core - with a velocity of $942 \mathrm{~km} / \mathrm{s}$. The estimated $\mathrm{C} 2$ masses with correction for the streamer brightness (which is equivalent to the mass $2 \cdot 10^{15} \mathrm{~g}$ ) are: for the frontal structure $6 \cdot 10^{15} \mathrm{~g}$, for the core $-4.6 \cdot 10^{15} \mathrm{~g}$. The total C3 CME mass at $13: 42\left(25 \mathrm{R}_{\odot}\right)$ is $1.5 \cdot 10^{16} \mathrm{~g}$.

To study the CME initiation we analyzed the EUV dimmings in 175 (SPIRIT) and $195 \AA$ (EIT) using earlier developed image pre-processing and dimming photometry procedures (Chertok et al. 2004, Chertok\&Grechnev 2005, Slemzin et al. 2006). The drop of intensity in the dimming area relative to the total solar flux before the eruption was about $7 \%$ in $195 \AA$ and $4.5 \%$ in $175 \AA$ bands. The intensity in the dimming area started to decrease at the time of the prominence activation and the maximum of X-ray flare, so we can conclude that all three processes: initiation of the CME frontal structure, initiation of the core and X-ray flare occurred simultaneously within the temporal resolution of the EUV observations $(12-15 \mathrm{~min})$.

\section{Acknowledgements}

This study was partly supported by Russian Foundation for Basic Research (grant 05-02-17415). SOHO is a project of international cooperation between ESA and NASA.

\section{References}

Aulanier, G. and B. Schmieder, 2002, Astron. Astrophys. 386, 1106

Chertok, I.M. et al. 2004, Astron. Rep. 48, 407

Chertok, I.M. \& Grechnev, V.V. 2005, Solar Phys. 229, 95

Gontikakis, et al. 1997, Solar Phys. 172, 189

Heinzel, P., B. Rompolt, 1987, Solar Phys. 110, 171

Heinzel, P. et al. 2003, ESA SP-535, 447

Romeuf, D. et al. 2006, Astron. Astrophys. (in press)

Slemzin, V. et al. 2006, Proc. IAUS 233 (in press) 